\title{
Nontargeted and targeted metabolomics approaches reveal the key amino acid alterations involved in multiple myeloma
}

\author{
Lingling Yue ${ }^{1}$, Pengyun Zeng ${ }^{1}$, Yanhong $\mathrm{Li}^{2}$, Ye Chai ${ }^{1}$, Chongyang Wu ${ }^{1}$, Bingren Gao ${ }^{\text {Corresp. } 3}$ \\ 1 Department of Hematology, Lanzhou University Second Hospital, Lanzhou, Gansu, China \\ 2 Institute of Hematology, Lanzhou University Second Hospital, Lanzhou, Gansu, China \\ 3 Department of Cardiac Surgery, Lanzhou University Second Hospital, Lanzhou, Gansu, China \\ Corresponding Author: Bingren Gao \\ Email address: bingrengao@foxmail.com
}

Purpose: Multiple myeloma (MM), a kind of malignant neoplasm of plasma cells in the bone marrow, is a refractory disease. Understanding the metabolism disorders and identification of metabolomics pathways as well as key metabolites will provide new insights for exploring diagnosis and therapeutic targets of MM. Methods: We conducted nontargeted metabolomics analysis of MM patients and normal controls (NC) using ultrahigh-performance liquid chromatography (UHPLC) combined with quadrupole time-of-flight mass spectrometry (Q-TOF-MS) in 40 cases of cohort 1 subjects. The targeted metabolomics analysis of amino acids using multiple reaction monitoring-mass spectrometry (MRM-MS) was also performed in 30 cases of cohort 1 and 30 cases of cohort 2 participants, to comprehensively investigate the metabolomics disorders of MM.

Results: The nontargeted metabolomics analysis in cohort 1 indicated that there was a significant metabolic signature change between MM patients and NC. The differential metabolites were mainly enriched in metabolic pathways related to amino acid metabolism, such as protein digestion and absorption, and biosynthesis of amino acids. Further, the targeted metabolomics analysis of amino acids in both cohort 1 and cohort 2 revealed differential metabolic profiling between MM patients and NC. We identified 12 and 14 amino acid metabolites with altered abundant in MM patients compared to NC subjects, in cohort 1 and cohort 2, respectively. Besides, key differential amino acid metabolites, such as choline, creatinine, leucine, tryptophan, and valine, may discriminate MM patients from NC. Moreover, the differential amino acid metabolites were associated with clinical indicators of MM patients. Conclusions: Our findings indicate that amino acid metabolism disorders are involved in MM. The differential profiles reveal the potential utility of key amino acid metabolites as diagnostic biomarkers of MM. The alterations in metabolome, especially the amino acid metabolome, may provide more evidences for elucidating the pathogenesis and development of MM. 
1 Nontargeted and targeted metabolomics approaches reveal the key amino acid alterations

\section{2 involved in multiple myeloma}

3 Running title: Metabolomics analysis of MM

4 Lingling Yue ${ }^{1}$, Pengyun Zeng ${ }^{1}$, Yanhong $\mathrm{Li}^{2}$, Ye Chai ${ }^{1}$, Chongyang Wu ${ }^{1}$, Bingren $\mathrm{Gao}^{3}$

$5{ }^{1}$ Department of Hematology, Lanzhou University Second Hospital, Lanzhou, Gansu, 730030,

6 China.

$7 \quad{ }^{2}$ Institute of Hematology, Lanzhou University Second Hospital, Lanzhou, Gansu, 730030, China.

$8{ }^{3}$ Department of Cardiac Surgery, Lanzhou University Second Hospital, Lanzhou, Gansu, 730030,

9 China.

10 Corresponding author: Bingren $\mathrm{Gao}^{3}$

11 Address: Department of Cardiac Surgery, Lanzhou University Second Hospital, No. 82

12 Cuiyingmen, Lanzhou, Gansu, 730030, China.

13 Email: bingrengao@foxmail.com

14 Tel: +86-13919367999

15 Fax: +86-0931-8942262 


\section{Abstract}

18 Purpose: Multiple myeloma (MM), a kind of malignant neoplasm of plasma cells in the bone

19 marrow, is a refractory disease. Understanding the metabolism disorders and identification of

20 metabolomics pathways as well as key metabolites will provide new insights for exploring

21 diagnosis and therapeutic targets of MM.

22 Methods: We conducted nontargeted metabolomics analysis of MM patients and normal 23 controls (NC) using ultra-high-performance liquid chromatography (UHPLC) combined with 24 quadrupole time-of-flight mass spectrometry (Q-TOF-MS) in 40 cases of cohort 1 subjects. The 25 targeted metabolomics analysis of amino acids using multiple reaction monitoring-mass 26 spectrometry (MRM-MS) was also performed in 30 cases of cohort 1 and 30 cases of cohort 2 27 participants, to comprehensively investigate the metabolomics disorders of MM.

28 Results: The nontargeted metabolomics analysis in cohort 1 indicated that there was a 29 significant metabolic signature change between MM patients and NC. The differential 30 metabolites were mainly enriched in metabolic pathways related to amino acid metabolism, such

31 as protein digestion and absorption, and biosynthesis of amino acids. Further, the targeted 32 metabolomics analysis of amino acids in both cohort 1 and cohort 2 revealed differential 33 metabolic profiling between MM patients and NC. We identified 12 and 14 amino acid 34 metabolites with altered abundant in MM patients compared to NC subjects, in cohort 1 and 35 cohort 2, respectively. Besides, key differential amino acid metabolites, such as choline, 36 creatinine, leucine, tryptophan, and valine, may discriminate MM patients from NC. Moreover, 37 the differential amino acid metabolites were associated with clinical indicators of MM patients.

38 Conclusions: Our findings indicate that amino acid metabolism disorders are involved in MM.

39 The differential profiles reveal the potential utility of key amino acid metabolites as diagnostic 
40 biomarkers of MM. The alterations in metabolome, especially the amino acid metabolome, may

41 provide more evidences for elucidating the pathogenesis and development of MM.

42 Key words: Multiple myeloma; metabolomics profiles; metabolic pathways; differential amino

43 acid metabolites; diagnostic biomarkers

\section{Introduction}

45 Multiple myeloma (MM) is a kind of malignant neoplasm characterized by the accumulation of 46 plasma cells in the bone marrow (Kocemba-Pilarczyk et al. 2018; Xiang et al. 2017). It is the

47 second most common hematologic malignancy (Tabata et al. 2020) and accounts for $1 \%$ of all 48 cancers diagnosed in the United States (Schecter \& Lentzsch 2013), with an annual incidence of 496.3 new cases per 100,000 individuals (Neuse et al. 2020). Due to the recurrent relapsing disease 50 course, MM is considered mostly incurable and requires various therapies (Harding et al. 2019).

51 To date, much progress has been made in the treatment strategy of MM. According to these 52 clinical trials (Fayers et al. 2011; Gay et al. 2011; Mateos 2010), the combination of diverse 53 drugs, such as proteasome inhibitors, immune-modulatory drugs, monoclonal antibodies, HDAC 54 inhibitors and individual CAR-T cell therapy, and autologous hemopoietic stem cell 55 transplantation with traditional drugs, such as corticosteroids, alkylating agents and 56 anthracyclines, has achieved significant clinical effects, but MM remains an incurable disease.

57 Despite considerable advances in treatment, the prognosis of $\mathrm{MM}$ is still very heterogeneous 58 (Schavgoulidze et al. 2021). Several reports have demonstrated the links between MM treatment 59 and its early diagnosis (Korde et al. 2015; Mateos et al. 2013). Therefore, a more comprehensive 60 understanding of MM features will aid in the design of an effective treatment strategy for this 61 disease. 
62 Accumulating evidence has confirmed that metabolic alterations are involved in myeloma cell

63 growth and drug resistance (Maiso et al. 2015; Pinto et al. 2020). Metabolomics analysis is a

64 comprehensive method of metabolites that can dynamically monitor the intermediate and final

65 products of biochemical reactions and has been widely used in cancer diagnosis, treatment, and

66 prognosis (Armitage \& Southam 2016; Cao et al. 2020; Kochanowski et al. 2021). By analyzing

67 the metabolic profiles of MM patients at diagnosis and after achieving complete remission, some

68 of the metabolic changes, such as glutamine, cholesterol, and lysine, have been observed,

69 suggesting the potential of metabolic profiles in identifying MM biomarkers or monitoring

70 response to treatment (Puchades-Carrasco et al. 2013). Studies have also revealed significant

71 alterations in amino acid, lipid and energy metabolism by analyzing the metabolomic plasma

72 profile of MM patients and $\mathrm{NC}$ and suggested the potential of cellular metabolic processes as

73 promising therapeutic targets in MM (Steiner et al. 2018). In addition, more studies have focused

74 on the indispensable mediator in the plasma microenvironment, amino acids. Glutamine (Cory \&

75 Cory 2006; Pochini et al. 2014) metabolism has proven to have close correlations with

76 hematopoietic malignancies. Du et al. reported that 23 metabolites changed significantly in MM

77 mainly by arginine, proline and glycerol phospholipid metabolic pathways (Du et al. 2018). Zaal

78 et al. demonstrated that bortezomib resistance in MM was associated with increased serine

79 synthesis (Zaal et al. 2017). These findings have confirmed the crucial role of some metabolites

80 and metabolic pathways in the pathogenesis of MM. However, metabolism encompasses the

81 generation of energy, the synthesis and breakdown of glucose, amino acids and fatty acids, and

82 oxidative phosphorylation. The metabolic changes in MM cells include the generation,

83 accumulation and inhibition of metabolites as well as metabolic shifts (El Arfani et al. 2018). 
84 Although previous findings have revealed some metabolic changes in MM, the key metabolites

85 and metabolic pathways in MM have not been fully investigated.

86 Metabolomics approaches can be nontargeted or targeted. Nontargeted metabolomics involves

87 global profiling of the metabolome and often provides more information than targeted

88 metabolomics. However, targeted metabolomics can quantify a select group of metabolites, such

89 as amino acids, which is more quantitative than nontargeted metabolomics (Zhang et al. 2016).

90 In the current study, we conducted nontargeted and targeted metabolomics of amino acids to

91 explore the metabolism disorders and identify metabolomics pathways as well as key differential

92 amino acid metabolites in MM patients. Our findings will provide new insight for elucidating the

93 possible mechanism of $\mathrm{MM}$ and exploring promising biomarkers or therapeutic targets for this

94 disease.

95 Materials and methods

96 Study design and patient recruitment

97 In this study, a total of 70 participants in two cohorts were recruited for metabolomic detection.

98 Patients with MM were collected from Lanzhou University Second Hospital, and NC subjects

99 were recruited through conventional physical examination in the hospital. MM patients were

100 diagnosed according to the criteria of International Myeloma Working Group (IMWG)

101 (Rajkumar. 2016). The International Staging System (ISS) was used to evaluate disease

102 progression (Loehrer. 2006). The patients with MM in the study were all newly diagnosed cases.

103 Subjects with immunodeficiency disease, medication use, smoking habits, or underlying chronic

104 disease were excluded from the study at the preliminary screening stage. All MM patients in this

105 study were not treated with radiotherapy or chemotherapy. Finally, in the cohort 1,40

106 participants including $20 \mathrm{MM}$ patients and $20 \mathrm{NC}$ subjects, were selected for nontargeted- and 
107 targeted-metabolomics. We also collected the information about clinical variables, including the 108 biochemical detection of serum albumin (ALB), interleukin 6 (IL-6), lactic dehydrogenase

109 (LDH), $\beta 2$-microglobulin (B2-MG), globulin (GLB), and serum creatinine (SCR) using the

110 Beckman automatic biochemistry analyzer (Beckman Chemistry Analyzer AU5800, Beckman

111 Coulter, Inc., USA) and Roche electrochemiluminescence system (Cobas e 801 Analyzer, Roche

112 Diagnostics $\mathrm{GmbH}$, Germany), for all the 40 participants in cohort 1 . The study of cohort 1

113 mainly contained two parts: 1) all 40 participants underwent nontargeted metabolomics analysis,

114 and 2) 30 participants (including $15 \mathrm{MM}$ patients and $15 \mathrm{NC}$ subjects) underwent targeted

115 metabolomics analysis. In the cohort 2, another 30 cases, including $15 \mathrm{MM}$ patients and $15 \mathrm{NC}$

116 subjects, were recruited as the validation group of the targeted metabolomics analysis. Figure 1

117 summarized the study design, and detailed information on the participants could be found in

118 Table 1 (cohort 1) and Supplementary Table 1 (cohort 2). This study was approved by the

119 Medical Ethics Committee of Lanzhou University Second Hospital (2018a-037). Written

120 informed consent was obtained from all participants.

121 Nontargeted metabolomics analysis using ultrahigh-performance liquid chromatography

122 (UHPLC) combined with quadrupole time-of-flight mass spectrometry (Q-TOF-MS)

\section{Sample collection and preparation}

124 Peripheral blood samples of all 40 participants in cohort 1, including $20 \mathrm{MM}$ patients and $20 \mathrm{NC}$

125 subjects, were collected, and serum samples were obtained by centrifugation. The samples were

126 then stored at $-80^{\circ} \mathrm{C}$ before further processing for UHPLC-Q-TOF/MS analysis.

\section{UHPLC-Q-TOF-MS analysis}

128 UHPLC analysis was performed with an Agilent 1290 Infinity LC UHPLC system.

129 Chromatographic separation was carried out at $25^{\circ} \mathrm{C}$ on a hydrophilic interaction 
130 chromatography (HILIC) column with a flow rate of $0.3 \mathrm{~mL} / \mathrm{min}$. The mobile phase consisted of

131 solvent $\mathrm{A}$ (water $+25 \mathrm{mM}$ ammonium acetate $+25 \mathrm{mM}$ ammonia) and solvent $\mathrm{B}$ (acetonitrile).

132 Gradient elution was performed as follows: $95 \%$ solvent B with $0-0.5 \mathrm{~min}$; the linear change of

133 solvent B was from $95 \%$ to $65 \%$ within $0.5-7 \mathrm{~min}$, from $65 \%$ to $40 \%$ with $7-8 \mathrm{~min}$, and

134 maintained at $40 \%$ for $8-9 \mathrm{~min}$; then, the linear change of solvent B was from $40 \%$ to $95 \%$

135 within 9-9.1 min and maintained at 95\% for 9.1-12 min. The samples were placed in an

136 automatic sampler at $4^{\circ} \mathrm{C}$ throughout the whole analysis. To avoid the influence of signal

137 fluctuation caused by instrument detection, continuous analysis of samples was carried out in

138 random order. Moreover, pooled quality control (QC) samples (generated by taking an equal

139 aliquot of all the samples included in the experiment) were run at the beginning of the sample

140 queue for column conditioning and every ten injections thereafter to assess inconsistencies that

141 are particularly evident in large batch acquisitions in terms of retention time drifts and variation

142 in ion intensity over time.

143 Q-TOF-MS analysis was conducted on an AB Triple TOF 6600 mass spectrometer (AB

144 SCIEX, Framingham, MA, USA). After HILIC chromatographic separation, the electrospray 145 ionization (ESI) source conditions were as follows: ion Source Gas1 (Gas1): 60 psi, Ion Source

146 Gas2 (Gas2): 60, Curtain gas (CUR): 30 , source temperature at $600^{\circ} \mathrm{C}$, ionSapary Voltage

147 Floating (ISVF): $\pm 5500 \mathrm{~V}$ (positive and negative model); TOF MS scan m/z range: 60-1000 Da,

148 product ion scan m/z range: $25-1000 \mathrm{Da}$, TOF MS scan accumulation time: $0.20 \mathrm{~s} / \mathrm{spectra}$, and

149 product ion scan accumulation time: $0.05 \mathrm{~s} /$ spectra. Information-dependent acquisition (IDA)

150 was used to obtain the secondary MS, as well as high sensitivity mode, declustering potential

151 (DP): $\pm 60 \mathrm{~V}$ (positive and negative modes), and collision energy: $35 \pm 15 \mathrm{eV}$. IDA was set as

152 exclude isotopes within $4 \mathrm{Da}$ and candidate ions to monitor per cycle: 6 . 


\section{Data processing and analysis}

154 The raw data were converted into. mzXML format files using the ProteoWizard converter tool

155 and then processed using XCMS software for peak alignment, retention time correction, and

156 peak area extraction. Then, the structures of metabolites were identified by comparing the

157 accuracy of $\mathrm{m} / \mathrm{z}$ values $(<25 \mathrm{ppm})$ and matching second-stage spectra with the laboratory's self-

158 built database (Applied Protein Technology Co., Ltd., Shanghai, China).

159 After data preprocessing by Pareto scaling, multidimensional data analyses were carried out,

160 including unsupervised principal component analysis (PCA), supervised partial least squares

161 discrimination analysis (PLS-DA), and orthogonal partial least squares discriminant analysis

162 (OPLS-DA). Single-dimensional statistical analyses, including Student's t-test and fold change

163 (FC) analysis, were conducted. The variable importance in the projection (VIP) value (>1) in the

164 PLS-DA model and P value analyzed by Student's t-test were combined to confirm the

165 significance of differential metabolites. Significant differential metabolites were obtained when

166 VIP $>1$ and $\mathrm{P}$ value $<0.05$, and differential metabolites were selected when VIP $>1$ and $0.05<$

$167 \mathrm{P}$ value $<0.1$. Moreover, Kyoto Encyclopedia of Genes and Genomes (KEGG) pathway

168 (http://www.genome.jp/kegg/) pathway enrichment analysis for significant differential

169 metabolites was conducted by the MetaboAnalyst online tool

170 (http://www.metaboanalyst.ca/MetaboAnalyst/) to investigate the metabolomics pathways.

171 Targeted metabolomics analysis of amino acids using multiple reaction monitoring-mass

172 spectrometry (MRM-MS)

\section{Sample collection and preparation}

174 Peripheral blood samples of 30 out of 40 participants in cohort 1 (including $15 \mathrm{MM}$ patients and

$17515 \mathrm{NC}$ subjects) and another 30 cases in cohort 2 (the validation group, including $15 \mathrm{MM}$ 
176 patients and $15 \mathrm{NC}$ subjects) were collected for targeted metabolomics analysis. Serum samples

177 were obtained by centrifuging at $1000 \times \mathrm{g}$ for $10 \mathrm{~min}$ and then stored at $-80^{\circ} \mathrm{C}$.

178 MRM-MS analysis

179 The analysis was performed with an Agilent 1290 Infinity LC UHPLC system. The mobile

180 phases were as follows: solvent A was $25 \mathrm{mM}$ ammonium formate $+0.08 \%$ formic acid (FA) in

181 water, and solvent B was $0.1 \%$ FA-acetonitrile. The sample was placed in an automatic sampler

182 at $4^{\circ} \mathrm{C}$, the column temperature was $40^{\circ} \mathrm{C}$, the flow rate was $250 \mu \mathrm{L} / \mathrm{min}$, and the injection

183 volume was $1 \mu \mathrm{L}$. Gradient elution was performed as follows: the linear change of solvent B was

184 from $90 \%$ to $70 \%$ within $0-12 \mathrm{~min}$; from $70 \%$ to $50 \%$ with $12-18 \mathrm{~min}$; from $50 \%$ to $40 \%$ within

$18518-25 \mathrm{~min}$, from $40 \%$ to $90 \%$ with $30-30.1 \mathrm{~min}$; and maintained at $90 \%$ for $30.1-37 \mathrm{~min}$. In the

186 sample queue, a QC sample was also set in a certain number of experimental samples at every

187 interval to test and evaluate the stability and repeatability of the system. MS analysis was carried

188 out in positive ionization mode on a 5500 QTRAP mass spectrometer (AB SCIEX, Framingham,

189 MA, USA). The conditions of the 5500 QTRAP ESI source were as follows: ion source

190 temperature, $500^{\circ} \mathrm{C}$; Gas1, $40 \mathrm{psi}$; Gas2, $40 \mathrm{psi}$; CUR, $30 \mathrm{psi}$; and ISVF, $5500 \mathrm{~V}$. MRM mode

191 was then used to detect the ion pairs.

192 Data processing and analysis

193 The chromatographic peak area and analyte retention time were extracted using Multiquant

194 software. Based on the standards of amino acids and their derivatives, the analyte retention time

195 was corrected, followed by identification of the metabolites. PLS-DA was also conducted to

196 explore the amino acids profiling and differential amino acid metabolites between groups. Score

197 scatter plots and loading plots were generated to visualize the separation of samples and

198 metabolites. MedCalc software (v19.0.4) was utilized for receiver operating characteristic curve 
199 (ROC) analysis to evaluate the diagnostic accuracy of amino acid metabolites. Moreover,

200 Spearman correlation analysis was performed with the statistical platform R package (v3.2.4).

201 KEGG pathway enrichment analysis for significant differential metabolites was also conducted

202 by the MetaboAnalyst online tool.

\section{Results}

204 Nontargeted metabolomics analysis using UHPLC-Q-TOF-MS

205 Multivariate statistical analysis

206 To analyze the metabolic changes between MM patients and NC, nontargeted metabolomics

207 analysis was carried out using UHPLC-Q-TOF-MS. As a result, the PCA score plots showed a

208 clear separation between MM patients and NC in both positive-ion and negative-ion modes

209 (Figure 2A). To further identify ion peaks that could possibly be used to differentiate the

210 metabolic profiles of MM patients and NC, the supervised PLS-DA model was conducted. The

211 results showed that MM patients were also separated from NC by the PLS-DA score plots in

212 both modes (Figure 2B). Consistent results were also obtained by the OPLS-DA score plots in

213 both modes (Figure 2C). Further permutation tests consisting of 200 permutations demonstrated

214 that the model was not overfitted (positive-ion model: $\mathrm{R} 2=(0.0,0.707), \mathrm{Q} 2=(0.0,-0.3673)$;

215 negative-ion model: $\mathrm{R} 2=(0.0,0.8331), \mathrm{Q} 2=(0.0,-0.4174)$; Figure 2D). These data indicated

216 that there was a significant metabolic change between MM patients and NC.

217 Analysis of differential metabolites between MM patients and NC

218 To compare differences in metabolites between MM patients and NC, differential metabolites

219 were screened out between MM patients and NC in both positive-ion and negative-ion modes

220 based on VIP $>1$ in the PLS-DA model and $\mathrm{P}<0.1$ analyzed by Student's t-test. The results

221 showed that a total of 62 differential metabolites were identified between MM patients and NC 
222 in positive-ion mode, of which 56 were significant $(\mathrm{P}<0.05)$. Similarly, 45 differential

223 metabolites were identified between MM patients and $\mathrm{NC}$ in negative-ion mode, of which 38

224 were significant $(\mathrm{P}<0.05)$ (Supplementary Table 2$)$. These data confirmed that there were

225 significant differences in metabolites between MM patients and NC.

226 Metabolic pathway analysis

227 To investigate the metabolomic pathways involved in MM development, the differential 228 abundant metabolites were enriched for the related metabolic pathway analysis using the

229 MetaboAnalyst online tool. As shown in Figure 3, differential metabolites were significantly 230 enriched in multiple metabolic pathways, such as protein digestion and absorption (including L231 alanine, L-glutamine, L-tryptophan, L-histidine, L-asparagine, L-valine, and L-isoleucine), ABC 232 transporters (including L-alanine, L-glutamine, L-histidine, L-valine, and L-isoleucine), and 233 biosynthesis of amino acids (L-alanine, L-glutamine, L-tryptophan, L-histidine, L-asparagine, L234 valine, L-citrulline, and L-isoleucine). Notably, these pathways were associated with amino acid 235 metabolism.

236 Targeted metabolomics analysis of amino acids using MRM-MS

237 Serum amino acid metabolites of MM patients and NC showed distinguished profiles

238 Previously, the results indicated that the altered metabolic profile of MM was mainly involved in 239 amino acid metabolomics features. We further conducted a target amino acid metabolomics 240 analysis to gain more insight into MM metabolism. Generally, in the cases of cohort 1, the PLS-

241 DA two-dimensional (2D) score plot showed clearly distinguished profiles between the MM and

242 NC groups (Figure 4A). Moreover, scatter loading plot analysis was carried out to evaluate

243 whether these differential amino acid metabolites accounted for the distinguished profiles. MM 244 patients exhibited higher levels of choline, creatinine, glutamate, and asparagine as well as lower 
245 levels of alanine/sarcosine, valine, tryptophan, and cystine than NC (Figure 4B). These data

246 suggested that altered amino acid profiles could discriminate MM patients from NC.

247 Expression of differential amino acid metabolites and the KEGG functional analysis

248 According to the metabolomics features, with the purpose of finding potential diagnostic

249 biomarkers or therapeutic targets for MM, we focused on the differential amino acid metabolites

250 in MM patients compared to those in NC. To further investigate the detailed variety degree of 251 each metabolite, we analyzed the intensity differences in a total of 28 amino acid metabolites.

252 Compared to NC, MM patients exhibited differential metabolic profiles. Overall, in the cases of 253 cohort 1 , there was a total of 12 significantly differential abundant amino acid metabolites in $254 \mathrm{MM}$ patients relative to $\mathrm{NC}(\mathrm{P}<0.05)$, including lysine, leucine, isoleucine, histidine, valine, 255 threonine, glutamine, tryptophan, choline, ornithine, creatinine, and alanine/sarcosine

256 (Supplementary Table 3). Furthermore, we validated these findings with the samples in cohort 257 2, and the results showed similar dysregulation trends with the findings of cohort 1 , that we 258 identified 14 significantly differential abundant amino acid metabolites in MM group 259 (Supplementary Table 4). Specifically, the high expression levels of creatinine (FC = 2.029, P $260<0.01$ in cohort $1 ; \mathrm{FC}=1.632, \mathrm{P}<0.05$ in cohort 2$)$ and choline $(\mathrm{FC}=1.508, \mathrm{P}<0.001$ in 261 cohort $1 ; \mathrm{FC}=1.234, \mathrm{P}=0.125$ in cohort 2) were found in $\mathrm{MM}$ patients in both cohort 1 and 262 cohort 2. Besides, since glutamate signaling has been reported involved in malignant disorders 263 (Willard \& Koochekpour 2013), we also examined glutamate levels and found slightly high 264 levels in $\mathrm{MM}$ patients $(\mathrm{FC}=1.186, \mathrm{P}=0.245$ in cohort $1 ; \mathrm{FC}=1.292, \mathrm{P}=0.346$ in cohort 2).

265 To determine the pathway impact of these differential amino acid metabolites, we performed 266 pathway classifications provided by KEGG analysis. Results indicated that the 12 differential 267 abundant metabolites in cohort 1 were significantly enriched in 22 metabolic pathways, such as 
268 aminoacyl-tRNA

biosynthesis,

valine/leucine/isoleucine

biosynthesis,

269 phenylalanine/tyrosine/tryptophan biosynthesis, D-glutamine/D-glutamate metabolism, and

270 histidine metabolism (Figure 4C, D).

271 Differential amino acid metabolites may discriminate MM patients from NC

272 We subsequently evaluated the performances of these differential abundant metabolites in

273 distinguishing MM from NC group. ROC analysis showed that, the amino acid metabolites

274 classifier could act as potential disease diagnostic biomarkers in both cohort 1 and cohort 2

275 (AUC $>0.7, \mathrm{P}<0.05$, Table 2 and Supplementary Figure 1). In MM patients of cohort 1, the

276 AUC values of two representative upregulated amino acid metabolites, choline and creatinine,

277 were 0.822 and 0.822 , respectively (Table 2). Similarly, the AUC of creatinine in the cohort 2

278 was 0.747 (Table 2). Meanwhile, the ROC analysis also showed good diagnostic values of the

279 downregulated amino acid metabolites in MM, such as leucine (AUC $=0.871$ in cohort 1 , AUC

$280=0.920$ in cohort 2$)$ and tryptophan $(\mathrm{AUC}=0.884$ in cohort $1, \mathrm{AUC}=0.902$ in cohort 2).

281 Specifically, we observed extremely high AUC values of the metabolite valine in both cohort 1

$282(\mathrm{AUC}=0.964)$ and cohort $2(\mathrm{AUC}=0.960)$. The results above suggested that these key

283 differential amino acid metabolites might be regarded as potential biomarkers for the diagnosis

284 or therapeutic targets of MM.

285 Differential amino acid metabolites were associated with MM phenotypes

286 To investigate the correlations between differential amino acid metabolites and MM-associated

287 phenotypes, we analyzed the correlations among 6 clinical indices/variables and 12 amino acid

288 metabolites that differed significantly in abundance between MM patients and NC in the cohort 1

289 (Figure 5). The clinical variables included serum albumin (ALB), interleukin 6 (IL-6), lactic

290 dehydrogenase (LDH), $\beta 2$-microglobulin (B2-MG), globulin (GLB), and serum creatinine (SCR). 
291 Among 12 amino acid metabolites whose abundances differed in MM patients, two mainly

292 correlation clusters were obtained. The cluster of upregulated components, creatinine and choline

293 in MM patients, was positively correlated with the levels of LDH, SCR, and B2-MG. Moreover,

294 in the downregulated amino acids cluster, 10 amino acid metabolites were negatively correlated

295 with the levels of IL-6 and GLB. Among these metabolites, the relationship between GLB and

296 valine/isoleucine/tryptophan/leucine/sarcosine exhibited high correlation coefficients (adjusted P

$297<0.01)$. These data suggested that the combination of clinical indices and amino acid metabolites

298 alterations facilitate a better understanding of MM progression.

299 Discussion

300 Metabolite disorder plays an essential role in cancer initiation and progression (La Vecchia \&

301 Sebastián 2020; Whisner \& Aktipis 2019). Metabolic changes in the tumor microenvironment

302 affect the effects of immunotherapy (Kouidhi et al. 2018). Therefore, a better understanding of

303 metabolic changes in the tumor microenvironment will improve the beneficial effects of

304 immunotherapy. Recently, with the development of metabolomics, more researchers have

305 focused on the clinical examination of metabolite variation, which might provide more predictors

306 and clues in disease diagnosis and treatment (Chen et al. 2019).

$307 \mathrm{MM}$, as a heterogenic disease with dynamic metabolic processes in bone marrow and its

308 microenvironment, has been reported to exhibit metabolic changes (El Arfani et al. 2018; Silva et

309 al. 2020). Despite this, the specific metabolic profiles in MM patients and potential clinical

310 biomarkers remain ambiguous and need to be further clarified. Metabolomics analysis is a

311 powerful means for investigating metabolic processes, revealing metabolic mechanisms and

312 identifying crucial biomarkers responsible for metabolic alteration. In the present study, we

313 conducted nontargeted metabolomics analysis using UHPLC-Q-TOF-MS and found a significant 
314 metabolic change between MM patients and NC. Differential metabolites could discriminate

315 MM patients and NC, which were significantly enriched in metabolic pathways related to amino

316 acid metabolism, such as protein digestion, and absorption and biosynthesis of amino acids.

317 These data suggested the key role of amino acid metabolism in MM. We then conducted the

318 targeted metabolomics analysis of amino acids using MRM-MS in two cohorts of participants

319 and found that serum amino acid metabolites of MM patients and NC showed distinguished

320 profiles. Compared to NC, MM patients exhibited higher levels of choline and creatinine as well

321 as lower levels of valine, tryptophan, leucine, etc. In another related study, the lipids profiles of

322 MM showed lower concentrations of phosphatidylcholine (PC), lysophosphatidylcholine (LPC)

323 and sphingomyelins (SM) (Silva et al. 2020). Besides, the concentration of essential amino acids,

324 especially tryptophan, was significantly decreased in MM cases (Silva et al. 2020), which was

325 consistent with our findings. Further ROC analysis in this study showed relatively good

326 diagnostic values of upregulated amino acid metabolites, including choline and creatinine, and

327 downregulated amino acid metabolites, such as leucine, tryptophan and valine. These results

328 suggested that the key differential amino acid metabolites could be used as promising biomarkers

329 for MM diagnosis. In addition, the clinical indicators were found to be related to altered amino

330 acid metabolomics in correlation analysis. MM patients were accompanied by elevation of

$331 \beta 2$-microglobulin ( $\beta 2-\mathrm{MG}$ ), and researchers found an inverse correlation between the

332 concentration of tryptophan and $\beta 2-\mathrm{MG}$ (Silva et al. 2020). In the current study, we also

333 observed a negative correlation between the differential metabolite tryptophan and $\beta 2$-MG.

334 Besides, the downregulated amino acids, including lysine, leucine, and valine, were also

335 negatively related with the high level of $\beta 2$-MG. Taken together, we hypothesized that the 
336 diagnostic value may be improved by combining examination of clinical indicators and amino 337 acid levels.

338 Amino acids and proteins have been shown to play central roles in cellular metabolism

339 (Rizzieri et al. 2019; Zhang et al. 2019). Amino acids are responsible for the formation of a 340 variety of components that are used for cell proliferation (Martinez-Outschoorn et al. 2017).

341 Notably, it has been reported that targeting amino acid metabolism may be a promising therapy

342 for cancer, suggesting the crucial role of amino acid metabolism in cancer (Bai et al. 2020; Boon

343 et al. 2020; Wang \& Zou 2020). Moreover, protein digestion and absorption is also an enriched

344 metabolism/digestive pathway involved in mechanisms of inhibitory effect of 3-O-kaempferol-3-

345 O-acetyl-6-O-(p-coumaroyl)- $\beta$-D-glucopyranoside (HK-11, a flavonoid compound) on MM cell

346 proliferation (Hou et al. 2020), implying the potential role of protein digestion and absorption

347 pathway in MM development. Our nontarget metabolomics analysis revealed that the differential

348 metabolites between MM patients and $\mathrm{NC}$ were significantly enriched in metabolic pathways

349 related to amino acid metabolism. It can therefore be speculated that amino acid metabolism is

350 involved in MM. Further targeted metabolomics analysis of amino acids showed that the

351 concentration of key metabolites was altered in $\mathrm{MM}$, and most of the amino acid metabolites was

352 downregulated. We highlight that these key metabolites may act as potential biomarkers for MM,

353 and that the supplementation of the disease phenotype-negatively related metabolic components

354 may help prevent or improve the prognosis of MM. Recent studies have also investigated the

355 proteomic alterations in the bone marrow interstitial fluid and serum samples, and identified

356 candidate proteins to be associated with drug resistance in MM patients (Chanukuppa et al. 2019;

357 Chanukuppa et al. 2021). The complex metabolite and lipid profiling of bone marrow plasma

358 could help differentiate patients with monoclonal gammopathy of undetermined significance 
359 (MGUS) from MM (Gonsalves et al. 2020). Therefore, the metabolic and proteomic detection

360 provides more evidences for the development of improved diagnosis and treatment of MM. In

361 the current study, we also conducted the targeted metabolomics analysis involving amino acids

362 in MM patients, and it's necessary to broaden the metabolic classes to find novel biomarkers in

363 the future.

364 In conclusion, our results reveal that amino acid metabolism disorder is involved in MM. Key

365 differential amino acid metabolites, such as choline, creatinine, leucine, tryptophan, and valine,

366 may discriminate MM patients from NC. The alteration in metabolism, especially amino acid

367 metabolism, may provide evidences for elucidating the pathogenesis of MM and lay a reference

368 for clinical diagnosis and therapy of this disease. However, there are still several limitations in

369 our research. Although we summarized differential amino acid metabolites and explored their

370 diagnostic value, more functional validations in vivo are still needed. Moreover, the sample size

371 was relatively small to make some general conclusions. More research is needed to

372 support the results and to investigate the underlying biological functions of the key amino

373 acid metabolites with large scale and mechanism study in the future. Besides, confounders

374 such as medication taken or smoking habits of the individuals can influence the metabolic

375 trend, comprehensive research involving the factors should be conducted in larger cohort

376 in future research.

377 Acknowledgments: None.

378 Funding: This work was supported by the Cuiying Scientific and Technological Innovation

379 Program of Lanzhou University Second Hospital, CY2017-MS13

380 Conflicts of Interest: The authors declare that they have no conflicts of interest. 
381 Data availability statement: The raw data of this study have been deposited in FigShare

382 (https://figshare.com/) with the link: https://doi.org/10.6084/m9.figshare.14904135.v1.

\section{References}

384 Armitage EG, and Southam AD. 2016. Monitoring cancer prognosis, diagnosis and treatment 385 efficacy using metabolomics and lipidomics. Metabolomics 12:1-15.

386 Bai M, Che Y, Lu K, and Fu L. 2020. Analysis of deubiquitinase OTUD5 as a biomarker and 387 therapeutic target for cervical cancer by bioinformatic analysis. PeerJ 8: e9146.

388 Boon R, Kumar M, Tricot T, Elia I, Ordovas L, Jacobs F, One J, De Smedt J, Eelen G, Bird M et 389 al. 2020. Amino acid levels determine metabolism and CYP450 function of hepatocytes and 390 hepatoma cell lines. Nat Commun 11:1393.

391 Cao G, Song Z, Hong Y, Yang Z, Song Y, Chen Z, Chen Z, and Cai Z. 2020. Large-scale 392 targeted metabolomics method for metabolite profiling of human samples. Anal Chim Acta 393 1125:144-151.

394 Chanukuppa V, Paul D, Taunk K, Chatterjee T, Sharma S, Kumar S, Santra MK, and Rapole S. 395 2019. XPO1 is a critical player for bortezomib resistance in multiple myeloma: A quantitative 396 proteomic approach. J Proteomics 209:103504.

397 Chanukuppa V, Taware R, Taunk K, Chatterjee T, Sharma S, Somasundaram V, Rashid F, 398 Malakar D, Santra MK, and Rapole S. 2021. Proteomic Alterations in Multiple Myeloma: A 399 Comprehensive Study Using Bone Marrow Interstitial Fluid and Serum Samples. Front Oncol $400 \quad 10: 566804$.

401 Chen Z, Li Z, Li H, and Jiang Y. 2019. Metabolomics: a promising diagnostic and therapeutic 402 implement for breast cancer. OncoTargets and therapy 12:6797.

403 Cory JG, and Cory AH. 2006. Critical roles of glutamine as nitrogen donors in purine and 404 pyrimidine nucleotide synthesis: asparaginase treatment in childhood acute lymphoblastic 405 leukemia. In Vivo 20:587-589.

406 Du H, Wang L, Liu B, Wang J, Su H, Zhang T, and Huang Z. 2018. Analysis of the metabolic 407 characteristics of serum samples in patients with multiple myeloma. Frontiers in pharmacology $4089: 884$.

409 El Arfani C, De Veirman K, Maes K, De Bruyne E, and Menu E. 2018. Metabolic features of 410 multiple myeloma. International journal of molecular sciences 19:1200. 
411 Fayers PM, Palumbo A, Hulin C, Waage A, Wijermans P, Beksaç M, Bringhen S, Mary J-Y,

412 Gimsing P, and Termorshuizen F. 2011. Thalidomide for previously untreated elderly patients

413 with multiple myeloma: meta-analysis of 1685 individual patient data from 6 randomized clinical

414 trials. Blood, The Journal of the American Society of Hematology 118:1239-1247.

415 Gay F, Larocca A, Wijermans P, Cavallo F, and Palumbo A. 2011. Complete response correlates

416 with long-term progression-free and overall survival in elderly myeloma treated with novel

417 agents: Analysis of 1175 patients. Blood 117:3025-3031.

418 Gonsalves WI, Broniowska K, Jessen E, Petterson XM, Bush AG, Gransee J, Lacy MQ, Hitosugi

419 T, and Kumar SK. 2020. Metabolomic and Lipidomic Profiling of Bone Marrow Plasma

420 Differentiates Patients with Monoclonal Gammopathy of Undetermined Significance from

421 Multiple Myeloma 10:10250.

422 Harding T, Baughn L, Kumar S, and Van Ness B. 2019. The future of myeloma precision 423 medicine: integrating the compendium of known drug resistance mechanisms with emerging 424 tumor profiling technologies. Leukemia 33:863-883.

425 Hou J, Qian J, Li Z, Gong A, Zhong S, Qiao L, Qian S, Zhang Y, Dou R, Li R et al. 2020. 426 Bioactive Compounds from Abelmoschus manihot L. Alleviate the Progression of Multiple 427 Myeloma in Mouse Model and Improve Bone Marrow Microenvironment. OncoTargets and 428 therapy 13:959-973.

429 Kocemba-Pilarczyk KA, Ostrowska B, Trojan S, Aslan E, Kusior D, Lasota M, Lenouvel C, and 430 Dulińska-Litewka J. 2018. Targeting the hypoxia pathway in malignant plasma cells by using 431 17-Allylamino-17-demethoxygeldanamycin. Acta Biochimica Polonica 65:101-109.

432 Kochanowski K, Sander T, Link H, Chang J, Altschuler SJ, and Wu LF. 2021. Systematic 433 alteration of in vitro metabolic environments reveals empirical growth relationships in cancer 434 cell phenotypes. Cell Rep 34:108647.

435 Korde N, Roschewski M, Zingone A, Kwok M, Manasanch EE, Bhutani M, Tageja N, 436 Kazandjian D, Mailankody S, and Wu P. 2015. Treatment with carfilzomib-lenalidomide437 dexamethasone with lenalidomide extension in patients with smoldering or newly diagnosed 438 multiple myeloma. JAMA oncology 1:746-754.

439 Kouidhi S, Ben Ayed F, and Benammar Elgaaied A. 2018. Targeting tumor metabolism: a new 440 challenge to improve immunotherapy. Frontiers in immunology 9:353. 
441 La Vecchia S, and Sebastián C. 2020. Metabolic pathways regulating colorectal cancer initiation 442 and progression. Seminars in cell \& developmental biology: Elsevier. p 63-70.

443 Loehrer PJ. 2006. International staging system for multiple myeloma. Yearbook of Oncology 444 2006:177-178.

445 Maiso P, Huynh D, Moschetta M, Sacco A, Aljawai Y, Mishima Y, Asara JM, Roccaro AM, 446 Kimmelman AC, and Ghobrial IM. 2015. Metabolic signature identifies novel targets for drug 447 resistance in multiple myeloma. Cancer research 75:2071-2082.

448 Martinez-Outschoorn UE, Peiris-Pages M, Pestell RG, Sotgia F, and Lisanti MP. 2017. Cancer 449 metabolism: a therapeutic perspective. Nature reviews Clinical oncology 14:11.

450 Mateos M-V, Hernández M-T, Giraldo P, de la Rubia J, de Arriba F, Corral LL, Rosiñol L, Paiva 451 B, Palomera L, and Bargay J. 2013. Lenalidomide plus dexamethasone for high-risk smoldering 452 multiple myeloma. New England Journal of Medicine 369:438-447.

453 Mateos M. 2010. Bortezomib, melphalan, and prednisone versus bortezomib, thalidomide, and 454 prednisone as induction therapy followed by maintenance treatment with bortezomib and 455 thalidomide versus bortezomib and prednisone in elderly patients with untreated multiple 456 myeloma. Lancet Oncology 11:934-941.

457 Neuse C J, Lomas O C, Schliemann C, Ghobrial IM. 2020. Genome instability in multiple 458 myeloma. Leukemia 34(11):1-11.

459 Pinto V, Bergantim R, Caires HR, Seca H, Guimaraes JE, and Vasconcelos MH. 2020. Multiple 460 Myeloma: Available Therapies and Causes of Drug Resistance. Cancers (Basel) 12.

461 Pochini L, Scalise M, Galluccio M, and Indiveri C. 2014. Membrane transporters for the special 462 amino acid glutamine: structure/function relationships and relevance to human health. Frontiers 463 in chemistry 2:61.

464 Puchades-Carrasco L, Lecumberri R, Martínez-López J, Lahuerta J-J, Mateos M-V, Prósper F, 465 San-Miguel JF, and Pineda-Lucena A. 2013. Multiple myeloma patients have a specific serum 466 metabolomic profile that changes after achieving complete remission. Clinical cancer research 467 19:4770-4779.

468 Rajkumar SV. 2016. Updated diagnostic criteria and staging system for multiple myeloma. Am. 469 Soc. Clin. Oncol. Educ. Book 36: 418-423. 
470 Rizzieri D, Paul B, and Kang Y. 2019. Metabolic alterations and the potential for targeting 471 metabolic pathways in the treatment of multiple myeloma. Journal of cancer metastasis and 472 treatment 5:26.

473 Schavgoulidze A, Cazaubiel T, Perrot A, Loiseau HA, Corre J. 2021. Multiple Myeloma: 474 Heterogeneous in Every Way. Cancers 13(6):1285.

475 Schecter J, and Lentzsch S. 2013. Multiple myeloma: defining the high-risk patient and 476 determining the optimal treatment strategy. Current hematologic malignancy reports 8:277-283.

477 Silva I, Levatti E, Pedroso AP, Marchioni DML, Carioca AAF, Colleoni GWB. 2020. 478 Biochemical phenotyping of multiple myeloma patients at diagnosis reveals a disorder of 479 mitochondrial complexes I and II and a Hartnup-like disturbance as underlying conditions, also 480 influencing different stages of the disease. Sci Rep 10(1): 21836.

481 Steiner N, Müller U, Hajek R, Sevcikova S, Borjan B, Jöhrer K, Göbel G, Pircher A, and 482 Gunsilius E. 2018. The metabolomic plasma profile of myeloma patients is considerably 483 different from healthy subjects and reveals potential new therapeutic targets. PloS one 484 13:e0202045.

485 Tabata M, Tsubaki M, Takeda T, Tateishi K, Maekawa S, Tsurushima K, Imano M, Satou T, 486 Ishizaka T, and Nishida S. 2020. Inhibition of HSP90 overcomes melphalan resistance through 487 downregulation of Src in multiple myeloma cells. Clinical \& Experimental Medicine 20:63-71.

488 Wang W, and Zou W. 2020. Amino Acids and Their Transporters in T Cell Immunity and 489 Cancer Therapy. Mol Cell 80:384-395.

490 Whisner CM, and Aktipis CA. 2019. The role of the microbiome in cancer initiation and 491 progression: how microbes and cancer cells utilize excess energy and promote one another's 492 growth. Current nutrition reports 8:42-51.

493 Willard SS, and Koochekpour S. 2013. Glutamate signaling in benign and malignant disorders: 494 current status, future perspectives, and therapeutic implications. International journal of 495 biological sciences 9:728.

496 Xiang T, Hu AX, Sun P, Liu G, Liu G, and Xiao Y. 2017. Identification of four potential 497 predicting miRNA biomarkers for multiple myeloma from published datasets. PeerJ 5:e2831.

498 Zaal EA, Wu W, Jansen G, Zweegman S, Cloos J, and Berkers CR. 2017. Bortezomib resistance 499 in multiple myeloma is associated with increased serine synthesis. Cancer \& metabolism 5:7. 
500 Zhang X, Yue D, Wang Y, Zhou Y, Liu Y, Qiu Y, Tian F, Yu Y, Zhou Z, and Wei W. 2019.

501 PASTMUS: mapping functional elements at single amino acid resolution in human cells. 502 Genome Biol 20:279.

503 Zhang X, Zhu X, Wang C, Zhang H, and Cai Z. 2016. Non-targeted and targeted metabolomics 504 approaches to diagnosing lung cancer and predicting patient prognosis. Oncotarget 7:63437. 
505

506

507

508

509

510

511

512

513

514

515

516

517

518

519

520

521

522

523

524

525

526

527

528

529

530

531

532

533

534

535

536

537

\section{Figure legends}

Figure 1. Overview of the study design and workflow. UHPLC-Q-TOF/MS: ultra-highperformance liquid chromatography (UHPLC) with quadrupole time-of-flight mass spectrometry (Q-TOF-MS); MRM-MS: multiple reaction monitoring-mass spectrometry; PCA: principal component analysis; PLS-DA partial-least squares discrimination analysis; OPLS-DA: orthogonal partial least squares discriminant analysis; KEGG: Encyclopedia of Genes and Genomes; ROC: receiver operating characteristic curve.

Figure 2. Score plots of supervised PCA, PLS-DA, and OPLS-DA analysis based on the combination of UHPLC-Q-TOF/MS data from MM patients and NC. (A) PCA score plots between MM patients and normal controls (NC) in both positive-ion and negative-ion modes. (B) PLS-DA score plots between MM patients and normal controls (NC) in both modes. (C) OPLSDA score plots between MM patients and normal controls (NC) in both modes. (D) Permutation tests consisting of 200 permutations demonstrated that the OPLS-DA model was not overfitted in either mode.

Figure 3. Metabolic pathway enrichment analysis of differential metabolites between MM patients and NC. Each related metabolic pathway is shown as a circle, whose size and color are based on the pathway impact value and the $\mathrm{P}$ value, respectively.

Figure 4. PLS-DA analysis based on MRM-MS data and functional pathway of key differential metabolites between MM and NC groups. (A, B) PLS-DA scores and loading plots between MM patients and normal controls based on the MRM-MS data. (C, D) Metabolic pathway enrichment analysis of differential amino acids metabolites between MM patients and NC. In (C), each related metabolic pathway is shown as a circle, whose size and color are based on the pathway impact value and the $\mathrm{P}$ value, respectively.

Figure 5. Correlation analysis of 12 differential amino acids identified from MM patients vs. NC with 6 clinical indicators. Numbers in the lower left panel: value of the correlation coefficient; symbols in the upper right panel: results of the significance test; *adjusted $\mathrm{P}<0.05$, **adjusted $\mathrm{P}<0.01$. ALB: albumin; LDH: lactic dehydrogenase; B2-MG: $\beta 2$-microglobulin.

Supplementary Figure 1. ROC curve analysis of the diagnostic value of differential amino acids for MM in cohort 1 (A) and cohort 2 (B). 


\section{Figure 1}

Overview of the study design and workflow.

UHPLC-Q-TOF/MS: ultra-high-performance liquid chromatography (UHPLC) with quadrupole time-of-flight mass spectrometry (Q-TOF-MS); MRM-MS: multiple reaction monitoring-mass spectrometry; PCA: principal component analysis; PLS-DA partial-least squares discrimination analysis; OPLS-DA: orthogonal partial least squares discriminant analysis; KEGG:

Encyclopedia of Genes and Genomes; ROC: receiver operating characteristic curve. 
20 patients with multiple myeloma (MM) and 20 normal controls (NC) as cohort 1

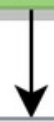

The peripheral blood samples were collected from the 40 participants

UHPLC-Q-TOF/MS
All 40 samples undergone the nonetargeted metabolomics

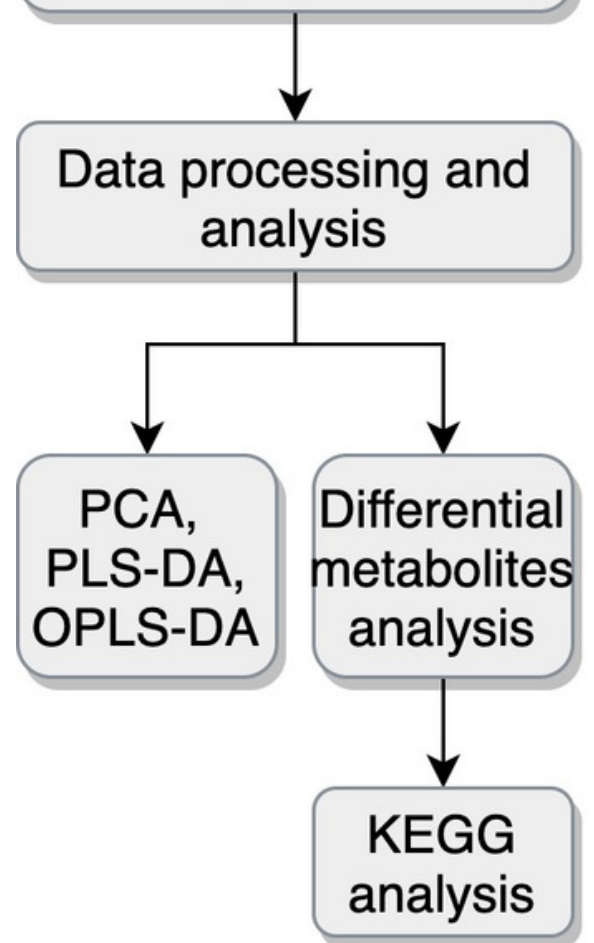

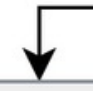

MRM-MS

30 out of 40 samples undergone the targeted metabolomics analysis

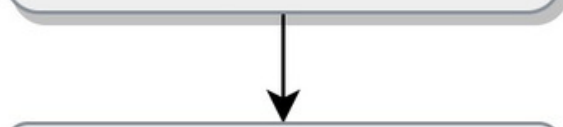

Data processing and analysis

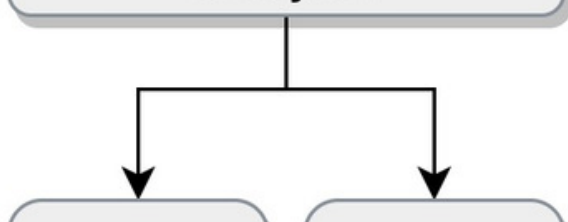

PLS-DA, load plot analysis

Amino acid analysis
15 cases of $\mathrm{MM}$ and 15 NCs as cohort 2

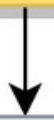

The peripheral blood samples were collected

MRM-MS

All 30 samples undergone the targeted metabolomics analysis

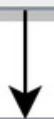

Data processing and analysis metabolites

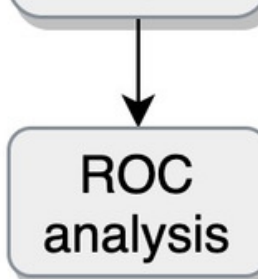




\section{Figure 2}

Score plots of supervised PCA, PLS-DA, and OPLS-DA analysis based on the combination of UHPLC-Q-TOF/MS data from MM patients and NC.

(A) PCA score plots between MM patients and normal controls (NC) in both positive-ion and negative-ion modes. (B) PLS-DA score plots between MM patients and normal controls (NC) in both modes. (C) OPLS-DA score plots between MM patients and normal controls (NC) in both modes. (D) Permutation tests consisting of 200 permutations demonstrated that the OPLS-DA model was not overfitted in either mode. 
A

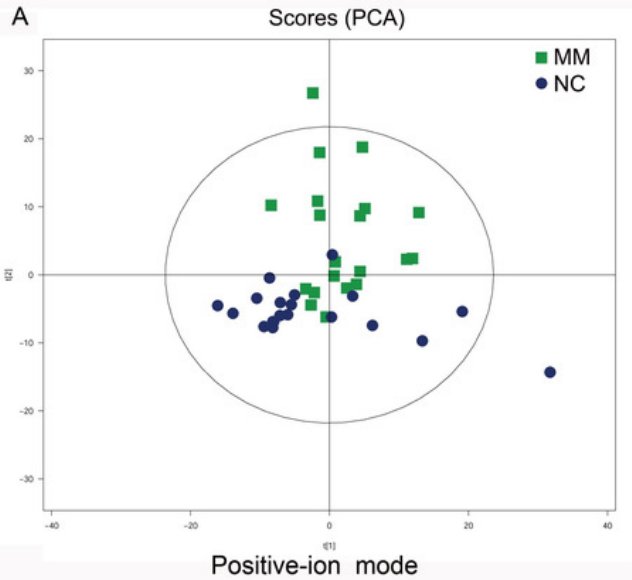

B

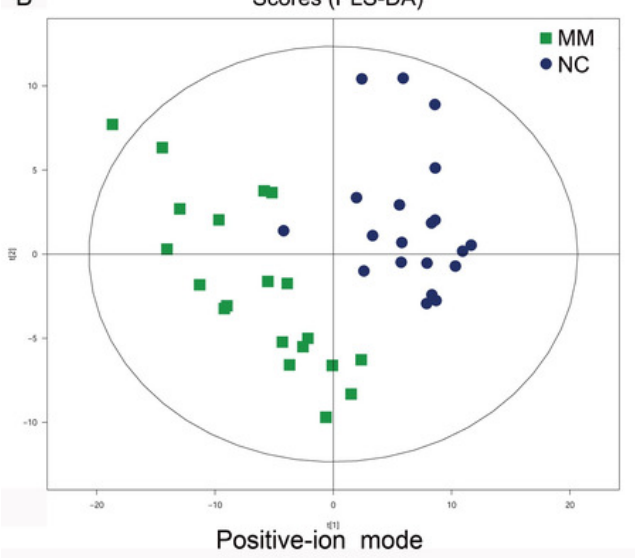

C

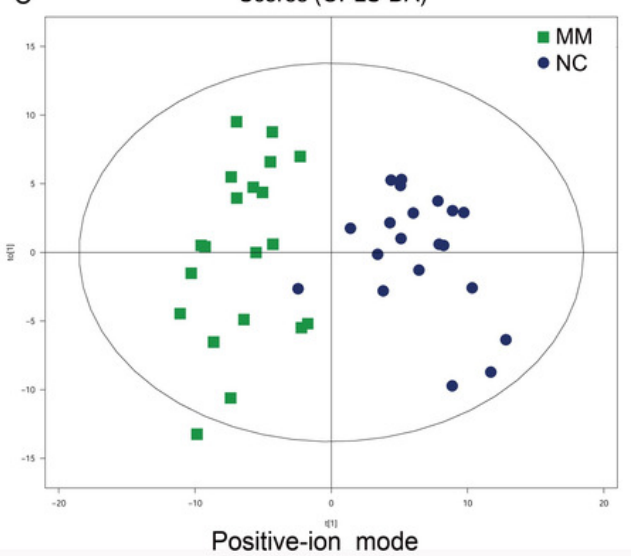

D

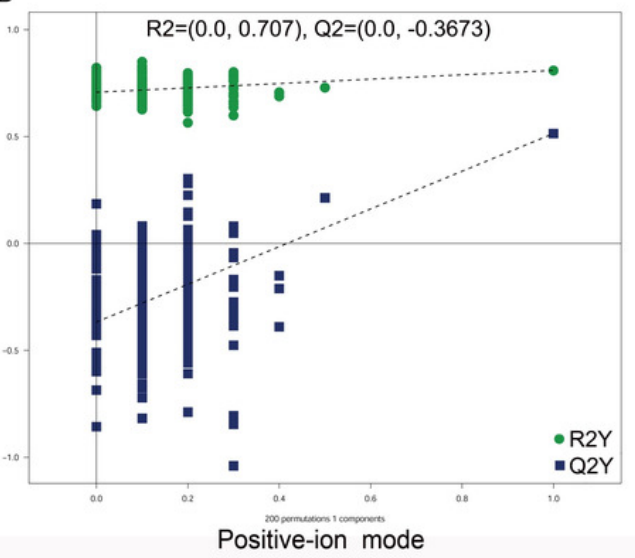

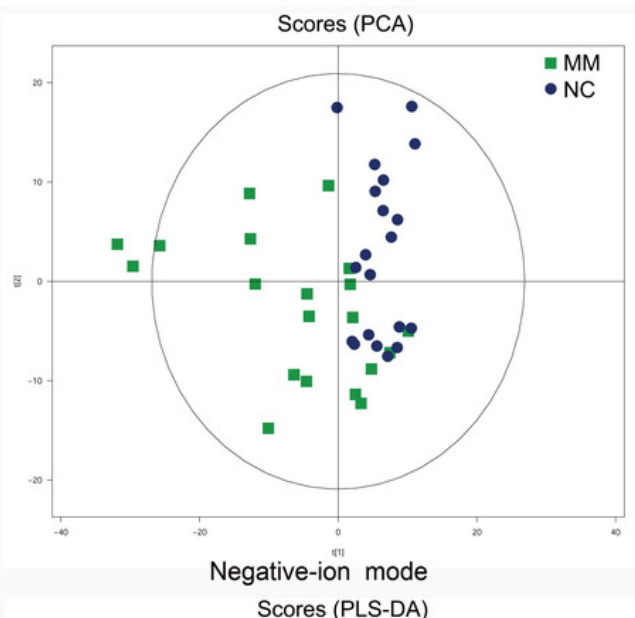

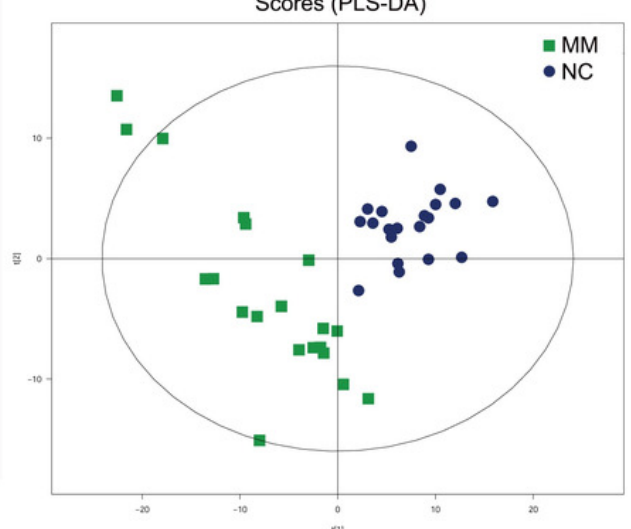

Negative-ion mode

Scores (OPLS-DA)

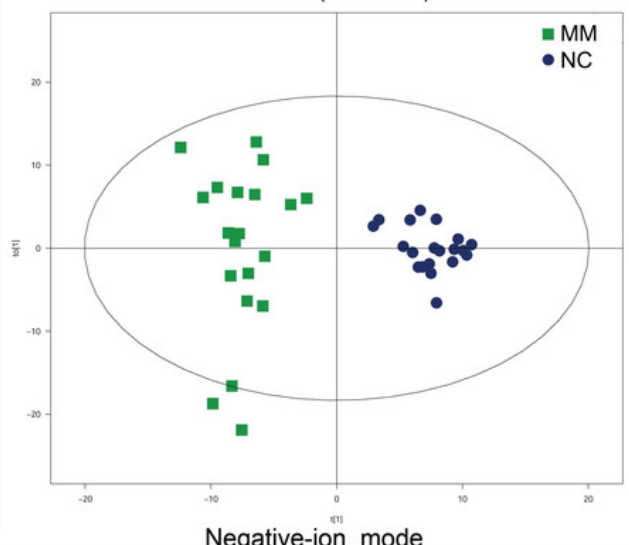

Negative-ion mode

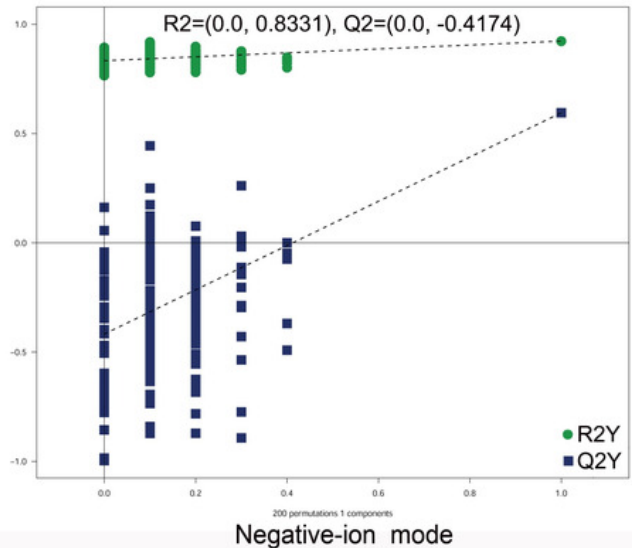


Figure 3

Metabolic pathway enrichment analysis of differential metabolites between MM patients and NC.

Each related metabolic pathway is shown as a circle, whose size and color are based on the pathway impact value and the $P$ value, respectively. 


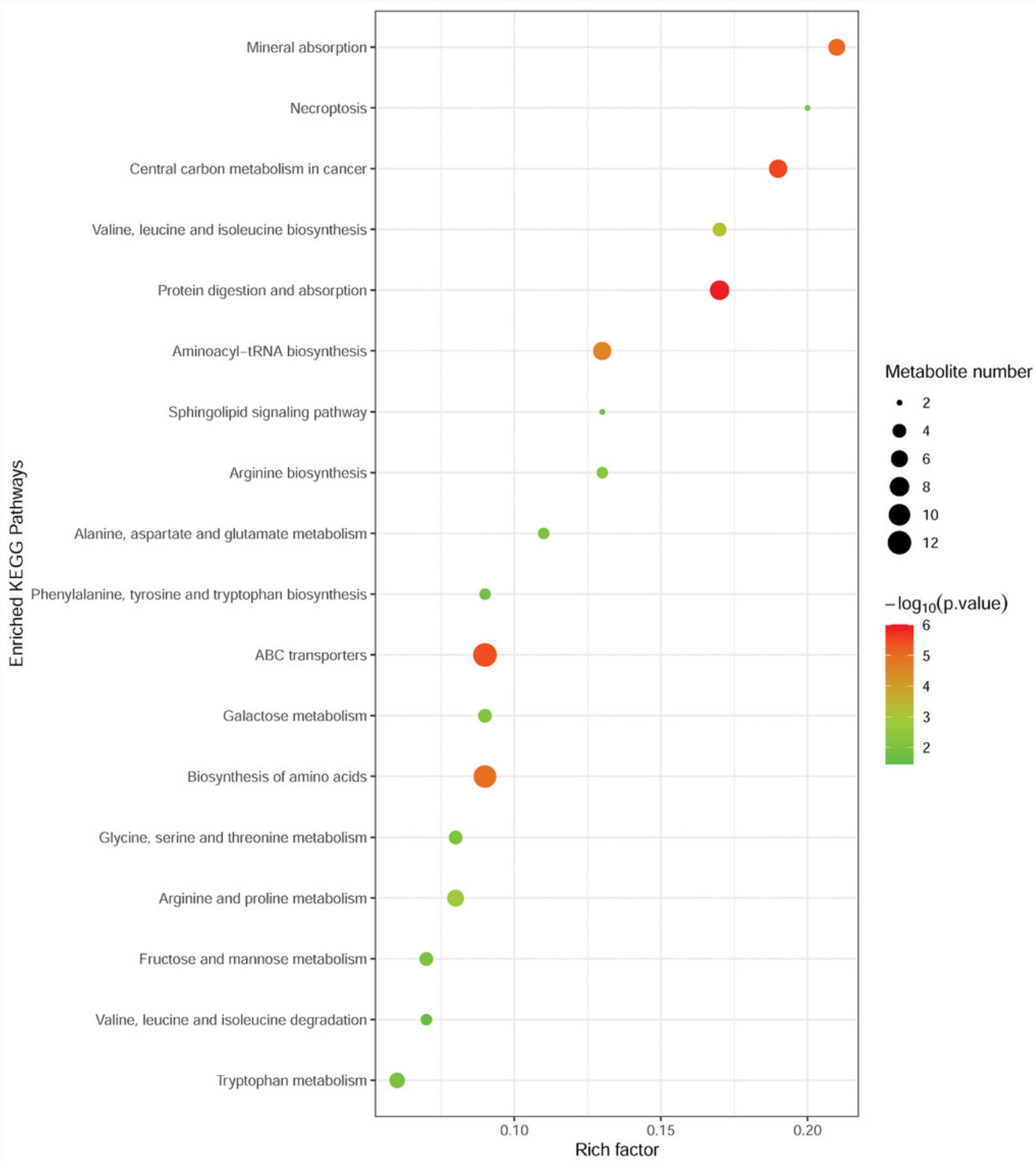


Figure 4

PLS-DA analysis based on MRM-MS data and functional pathway of key differential metabolites between MM and NC groups.

(A, B) PLS-DA scores and loading plots between MM patients and normal controls based on the MRM-MS data. (C, D) Metabolic pathway enrichment analysis of differential amino acids metabolites between MM patients and NC. In (C), each related metabolic pathway is shown as a circle, whose size and color are based on the pathway impact value and the $\mathrm{P}$ value, respectively.
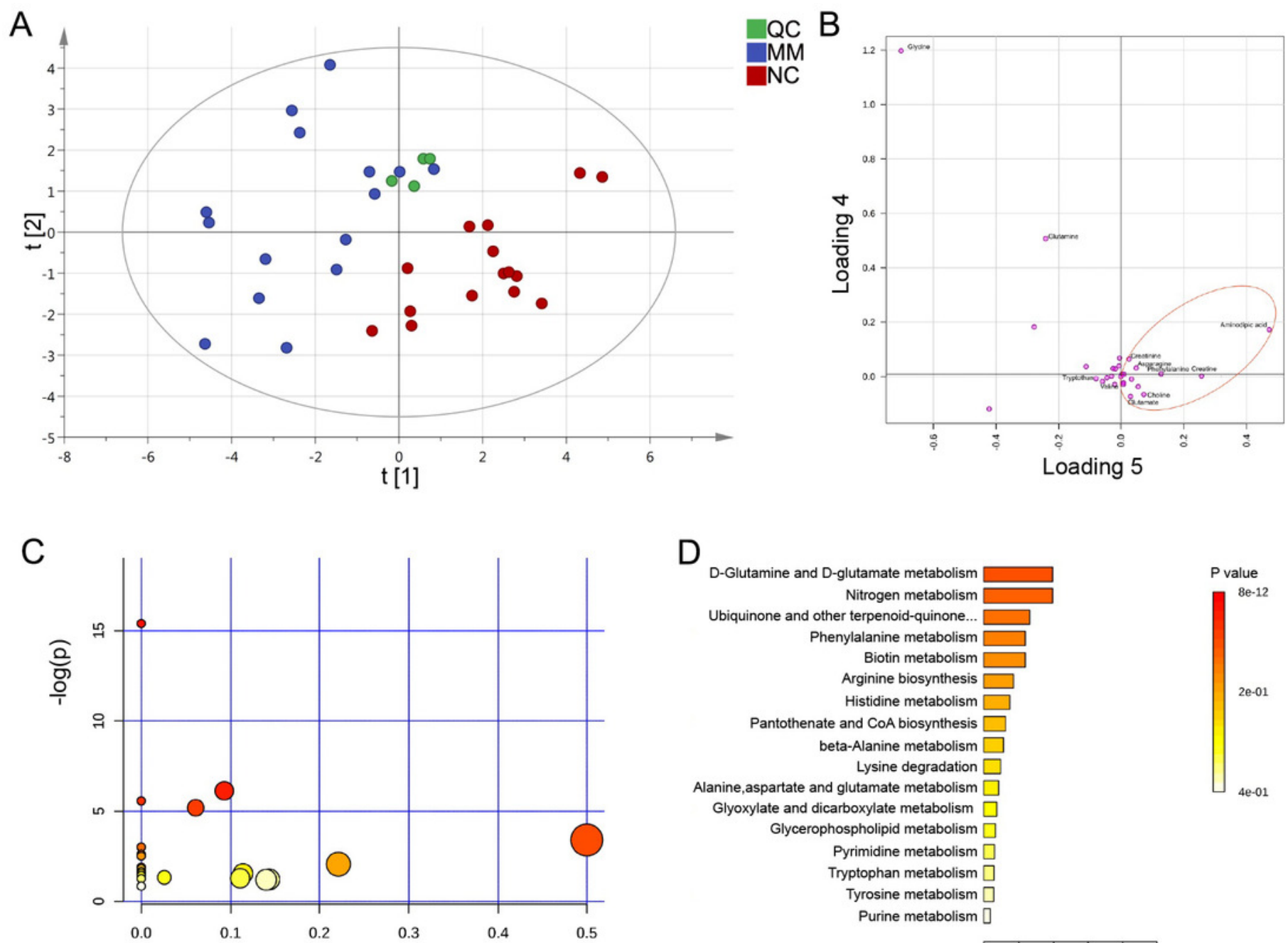

$\mathrm{D}$

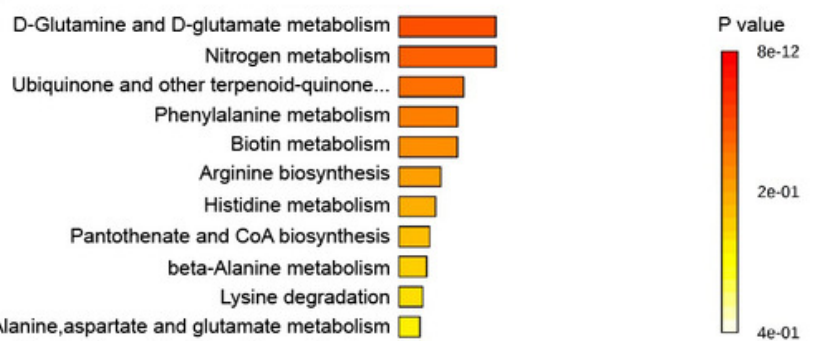

Alanine,aspartate and glutamate metabolism $\square$

Glyoxylate and dicarboxylate metabolism $\square$

Glycerophospholipid metabolism $\square$

Pyrimidine metabolism $\square$

Tryptophan metabolism $\square$

Tyrosine metabolism $\square$

Purine metabolism [?

Pathway impact

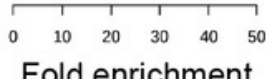

Fold enrichment 


\section{Figure 5}

Correlation analysis of 12 differential amino acids identified from MM patients vs. NC with 6 clinical indicators.

Numbers in the lower left panel: value of the correlation coefficient; symbols in the upper

right panel: results of the significance test; *adjusted $\mathrm{P}<0.05$, ${ }^{* *}$ adjusted $\mathrm{P}<0.01$. ALB: albumin; LDH: lactic dehydrogenase ; B2-MG : $\beta 2$-microglobulin . 


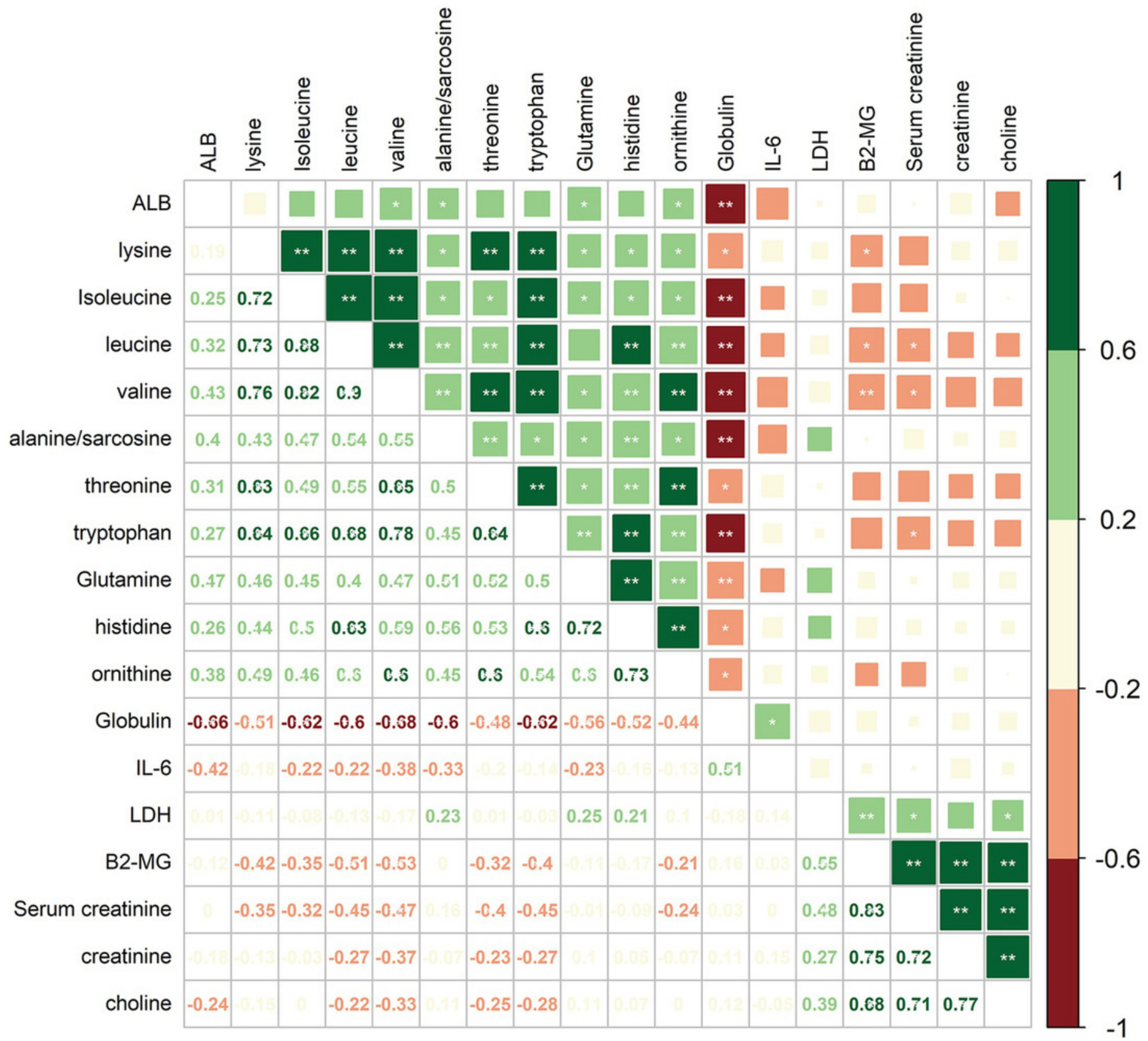




\section{Table $\mathbf{1}$ (on next page)}

Clinical characteristics of the 40 participants in cohort 1

Abbreviations: ISS: International Staging System; ALB: albumin, reference range: 40-55; IL-6: interleukin 6, reference range: 0.00-5.30; LDH: lactic dehydrogenase, reference range:

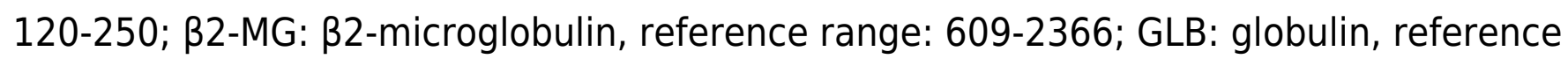
range: 20-40; SCR: serum creatinine, reference range: 41.0-73.0. Compared with the NC group, "Significant association with $\mathrm{P}<0.05$, " Significant association with $\mathrm{P}<0.01$, ${ }^{\Delta}$ Significant association with $\mathrm{P}<0.001$. 
Table 1. Clinical characteristics of the 40 participants in cohort 1

\begin{tabular}{|c|c|c|c|c|c|c|c|c|c|}
\hline Group & Age & Sex & ISS & $\operatorname{ALB}(g / L)$ & IL-6 (pg/mL) & $\mathrm{LDH}(\mathrm{U} / \mathrm{L})$ & $\beta 2-\mathrm{MG}(\mathrm{ng} / \mathrm{mL})$ & GLB $(g / L)$ & $\operatorname{SCR}(\mu \mathrm{mol} / \mathrm{L})$ \\
\hline $\begin{array}{l}\text { Normal control } \\
(\mathrm{NC}, \mathrm{n}=20)\end{array}$ & $55.10 \pm 10.43$ & $\begin{array}{l}\text { Female }(\mathrm{n}=8) \\
\text { Male }(\mathrm{n}=12)\end{array}$ & --- & $42.16 \pm 6.12$ & $4.21 \pm 1.98$ & $183.00 \pm 66.28$ & $1037.05 \pm 423.28$ & $27.62 \pm 6.51$ & $67.88 \pm 33.47$ \\
\hline $\begin{array}{l}\text { Multiple myeloma } \\
(\mathrm{MM}, \mathrm{n}=20)\end{array}$ & $54.90 \pm 11.29$ & $\begin{array}{l}\text { Female }(\mathrm{n}=8) \\
\text { Male }(\mathrm{n}=12)\end{array}$ & $\begin{array}{c}\text { ISS I }(\mathrm{n}=4) \\
\text { 1SS II }(\mathrm{n}=6) \\
\text { ISS III }(\mathrm{n}=10)\end{array}$ & $37.89 \pm 9.08$ & $12.24 \pm 14.72^{*}$ & $241.20 \pm 112.80$ & $10846.45 \pm 10615.85^{\Delta}$ & $49.56 \pm 26.42^{\Delta}$ & $243.75 \pm 229.60^{\#}$ \\
\hline
\end{tabular}

2 Abbreviations: ISS: International Staging System; ALB: albumin, reference range: 40-55; IL-6: interleukin 6, reference range: 0.003 5.30; LDH: lactic dehydrogenase, reference range: 120-250; $\beta 2-\mathrm{MG}$ : $\beta 2$-microglobulin, reference range: 609-2366; GLB: globulin,

4 reference range: 20-40; SCR: serum creatinine, reference range: 41.0-73.0. Compared with the NC group, *Significant association 5 with $\mathrm{P}<0.05$, " Significant association with $\mathrm{P}<0.01,{ }^{\Delta}$ Significant association with $\mathrm{P}<0.001$. 


\section{Table 2 (on next page)}

Clinical characteristics of the 40 participants in cohort 1

Abbreviations: ISS: International Staging System; ALB: albumin, reference range: 40-55; IL-6: interleukin 6, reference range: 0.00-5.30; LDH: lactic dehydrogenase, reference range:

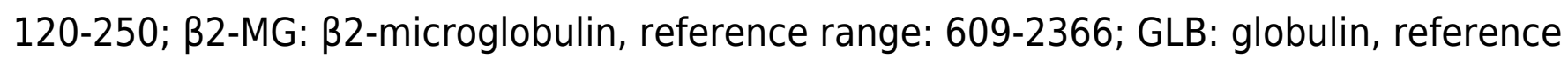
range: 20-40; SCR: serum creatinine, reference range: 41.0-73.0. Compared with the NC group, "Significant association with $\mathrm{P}<0.05$, " Significant association with $\mathrm{P}<0.01$, ${ }^{\Delta}$ Significant association with $\mathrm{P}<0.001$. 
Table 1. Clinical characteristics of the 40 participants in cohort 1

\begin{tabular}{|c|c|c|c|c|c|c|c|c|c|}
\hline Group & Age & Sex & ISS & $\operatorname{ALB}(\mathrm{g} / \mathrm{L})$ & IL-6 $\underline{(\mathrm{pg} / \mathrm{mL})}$ & $\mathrm{LDH}_{\underline{(\mathrm{U} / \mathrm{L})}}$ & $\beta 2-\mathrm{MG}_{(\mathrm{ng} / \mathrm{mL})}$ & $\operatorname{GLB}(\underline{g} / \mathrm{L})$ & $\operatorname{SCR}(\mu \mathrm{mol} / \mathrm{L})$ \\
\hline $\begin{array}{l}\text { Normal control } \\
(\mathrm{NC}, \mathrm{n}=20)\end{array}$ & $55.10 \pm 10.43$ & $\begin{array}{l}\text { Female }(\mathrm{n}=8) \\
\text { Male }(\mathrm{n}=12)\end{array}$ & --- & $42.16 \pm 6.12$ & $4.21 \pm 1.98$ & $183.00 \pm 66.28$ & $1037.05 \pm 423.28$ & $27.62 \pm 6.51$ & $67.88 \pm 33.47$ \\
\hline $\begin{array}{l}\text { Multiple myeloma } \\
(\mathrm{MM}, \mathrm{n}=20)\end{array}$ & $54.90 \pm 11.29$ & $\begin{array}{l}\text { Female }(\mathrm{n}=8) \\
\text { Male }(\mathrm{n}=12)\end{array}$ & $\begin{array}{c}\text { ISS I }(\mathrm{n}=4) \\
1 \mathrm{SS} \text { II }(\mathrm{n}=6) \\
\text { ISS III }(\mathrm{n}=10)\end{array}$ & $37.89 \pm 9.08$ & $12.24 \pm 14.72^{*}$ & $241.20 \pm 112.80$ & $10846.45 \pm 10615.85^{\Delta}$ & $49.56 \pm 26.42^{\Delta}$ & $243.75 \pm 229.60^{\#}$ \\
\hline
\end{tabular}

2 Abbreviations: ISS: International Staging System $;$, ALB: albumin, reference range: 40-55; IL-6: interleukin 6, reference range: 0.00-

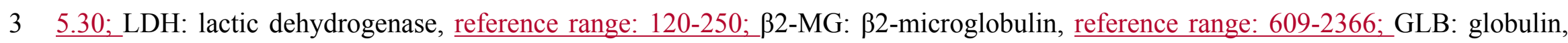
4 reference range: 20-40; SCR: serum creatinine, reference range: 41.0-73.0. Compared with the NC group, *Significant association 5 with $\mathrm{P}<0.05$, ${ }^{\text {}}$ Significant association with $\mathrm{P}<0.01$, ${ }^{\Delta}$ Significant association with $\mathrm{P}<0.001$. 


\section{Table 3 (on next page)}

Performance of differential metabolites in distinguishing MM from NC group 
Table 2. Performance of differential metabolites in distinguishing MM from NC group

cohort 1 metabolites sensitivity specificity AUC

\begin{tabular}{|c|c|c|c|c|c|c|c|c|c|}
\hline \multicolumn{5}{|c|}{ cohort 1} & \multicolumn{5}{|c|}{ cohort 2} \\
\hline metabolites & sensitivity & specificity & $\mathbf{A U C}$ & $\mathbf{P}$ & metabolites & sensitivity & specificity & $\mathbf{A U C}$ & $\mathbf{P}$ \\
\hline alanine/sarcosine & 60.0 & 93.3 & 0.751 & 0.008 & alanine/sarcosine & 66.7 & 93.3 & 0.773 & 0.003 \\
\hline choline & 66.7 & 100.0 & 0.822 & $<0.001$ & creatinine & 73.3 & 86.7 & 0.747 & 0.012 \\
\hline creatinine & 66.7 & 100.0 & 0.822 & $<0.001$ & Glutamine & 66.7 & 86.7 & 0.773 & 0.003 \\
\hline Glutamine & 100.0 & 60.0 & 0.778 & 0.002 & histidine & 80.0 & 86.7 & 0.907 & $<0.001$ \\
\hline histidine & 80.0 & 80.0 & 0.778 & 0.002 & Isoleucine & 60.0 & 93.3 & 0.840 & $<0.001$ \\
\hline Isoleucine & 73.3 & 80.0 & 0.782 & 0.001 & leucine & 86.7 & 86.7 & 0.920 & $<0.001$ \\
\hline leucine & 93.3 & 73.3 & 0.871 & $<0.001$ & lysine & 40.0 & 100.0 & 0.724 & 0.017 \\
\hline lysine & 40.0 & 100.0 & 0.720 & 0.024 & ornithine & 46.7 & 100.0 & 0.769 & 0.002 \\
\hline ornithine & 66.7 & 80.0 & 0.782 & 0.001 & serine & 80.0 & 73.3 & 0.738 & 0.012 \\
\hline threonine & 93.3 & 73.3 & 0.853 & $<0.001$ & taurine & 86.7 & 60.0 & 0.764 & 0.003 \\
\hline tryptophan & 73.3 & 93.3 & 0.884 & $<0.001$ & threonine & 93.3 & 66.7 & 0.827 & $<0.001$ \\
\hline valine & 86.7 & 93.3 & 0.964 & $<0.001$ & tryptophan & 73.3 & 93.3 & 0.902 & $<0.001$ \\
\hline & & & & & tyrosine & 80.0 & 66.7 & 0.769 & 0.002 \\
\hline & & & & & valine & 86.7 & 93.3 & 0.960 & $<0.001$ \\
\hline
\end{tabular}

cohort 2 